\title{
CpG-specific methylation at rheumatoid arthritis diagnosis as a marker of treatment response
}

\author{
"In rheumatoid arthritis, there are now a wide array of biological \\ agents that appear effective at population level; however, at the \\ individual level, only some respond and even less achieve clinical \\ remission."
}

First draft submitted: 17 January 2017; Accepted for publication: 2 February 2017; Published online: 27 April 2017

Keywords: DMARDs • DNA methylation • T lymphocytes

\section{Epigenetic mechanisms in rheumatoid arthritis pathogenesis}

It is now well established that aberrant epigenetic profiles significantly contribute to the pathogenesis of a vast number of disease states including rheumatoid arthritis (RA). $\mathrm{RA}$ is an autoimmune disease that results in immune activation, autoantibody and inflammatory cytokine production, and the destruction of cartilage leading to deformity and disability. This is a common disease with a prevalence of around 1\%.

In synovial fibroblasts, both global and gene-specific hypomethylation have been reported in LINE-1 [1] and CXCL12 [2] respectively. In addition, Nakano et al. [3] have shown that an abundance of differentially methylated regions exists across the genome of RA patients. Even differential methylation of a single CpG site in the IL- 6 gene may contribute to the inflammatory state associated with RA [4]. Another study has shown that loss of DNMT1 activity leading to aberrant $\mathrm{CpG}$ methylation at the MMP13 gene causes increased MMP13 expression and subsequently collagen degradation in the cartilage [5]. While it is certainly of interest to understand and map the epigenetic changes that are causative of diseases such as RA, the use of contemporary DNA methylation-profiling technologies may also be utilized to influence patient treatment.

\section{Treatment response based on epigenetic biomarkers}

In the context of cancer treatment, methylation status of a number of genes has been linked with drug sensitivity in a vast number of cancer/tumor types [6]. Furthermore, it has also recently been hypothesized that DNA methylation may alter an individual's responsiveness to psychiatric drugs, possibly due to variations in serotonin synthesis genes [7]. Therefore, investigation into the effects of differential methylation on the efficacy of treatments in other common diseases appears worthwhile. In RA, there are now a wide array of biological agents that appear effective at population level; however, at the individual level, only some respond and even less achieve clinical remission. The trial and error approach of current drug treatment is both expensive and cumbersome. In the rheumatology field, therefore, a lot can be learned from how cancer researchers are utilizing epigenetic biomarkers.

With this in mind, Glossop et al. [8] recently reported some unique and interesting findings; 46 patients with suspected RA, who were not receiving disease-modifying antirheumatic drug treatment, were recruited. Upon diagnosis, one or more diseasemodifying antirheumatic drugs (methotrexate, hydroxychloroquine or sulphasalazine) were prescribed, and disease activity quanti-

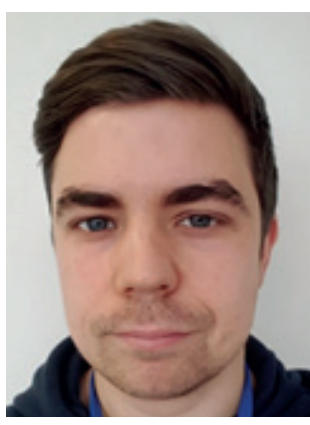

Steven Horsburgh Department of Applied Sciences, Northumbria University, Newcastle upon Tyne, UK

Marzena Ciechomska National Institute of Geriatrics, Rheumatology \& Rehabilitation, Warsaw, Poland

Steven O'Reilly

Author for correspondence: Department of Applied Sciences, Northumbria University, Newcastle upon Tyne, UK

steven.oreilly@northumbria.ac.uk 
fied 3 and 6 months later. Genome-wide methylation profiling of T-lymphocyte DNA at baseline revealed that $21 \mathrm{CpG}$ sites exhibited differences in methylation between treatment responders and nonresponders, four of which remained statistically significant following Bonferonni adjustment. Most notably, two CpG sites in the ADAMTSL2 and BTN3A2 genes were strongly associated with treatment response; at baseline, 28 of 29 of responders possessed this specific methylation pattern at these two sites. These data are particularly powerful given that long-term outcome is associated with the response to first treatment [9]. Furthermore, a more immediate dose adjustment based on likely treatment responsiveness would not only reduce costs and drug wastage, but potentially attenuate the time frame by which the patient would experience optimal treatment and thereby potentially prevent irreversible joint damage, as we know there is a 'window of opportunity' in RA after which treatment appears ineffective.

\section{"Various therapeutic miRs are now in preclinical and clinical trials, and are therefore, likely to represent important treatment options for a number of conditions in future.}

Use of the extensive HumanMethylation450k beadchip, validation of candidates using sodium bisulfite pyrosequencing, in addition to robust statistical measures, ensured that a large portion of the genome was covered using reliable techniques with low probability of type 1 errors. While the cost and coverage of the HumanMethylation450k beadchip should not be trivialized, further research of this nature should incorporate the very latest in microarray technology. In particular, the recently developed HumanMethylationEPIC beadchip has been expanded to include over 850,000 methylation sites. Again, we are aware of the difficulties surrounding participant recruitment and research concordance; however, larger sample sizes would enhance the robustness of the findings and could possibly identify further $\mathrm{CpG}$ sites associated with treatment response. It must be noted that the authors themselves acknowledge this. Additionally, while the authors state that short-term stimuli minimally affect DNA methylation, studies have shown that promoter methylation of a number of genes can be altered by a single bout of exercise [10,11], albeit within skeletal muscle and not lymphocytes. We propose, therefore, that a more extensive panel of baseline variables including habitual methyl-donor consumption through the diet and exercise, would provide even more robust data in larger populations. Overall, we congratulate the authors of this paper on highlighting a promising line of investigation which could significantly aid in the effectiveness and personalization of RA treatment.

\section{Potential systemic sclerosis biomarkers \& treatment}

Given these data and the relatively well-established role of differentially methylated drug metabolism enzyme genes in modification of the responsiveness to drug treatment, it is plausible that DNA methylation may also serve as a biomarker of treatment response in other rheumatic conditions associated with aberrant epigenetic profiles such as systemic sclerosis (SSc) [12,13].

The aforementioned differential methylation of a CpG site in the ADAMTSL2 gene, which is involved in TGF- $\beta$ regulation, could be postulated to also influence SSc treatment responses due to the central role of aberrant TGF- $\beta$ expression in SSc pathogenesis [14]. Furthermore, methotrexate, in its role as an immunosuppressant, is also used to treat SSc, which provides support to the possibility that SSc patients may also benefit from personalized treatment based on methylation-based predictions. Of course, prospective cohort studies would need to be conducted in order to confirm or refute such a supposition.

Gras et al. [15] reported that TGF- $\beta$ may be linked with fibrosis via miRNA (miR)-dependent mechanisms; elevated miR-145 attenuates KLF4 which in turn augments $\alpha$-SMA. The miR-29 family has also been implicated in SSc $[16,17]$, while miR-155 is thought to be a promising therapeutic target for arthritis [18]. Various therapeutic miRs are now in preclinical and clinical trials, and are therefore, likely to represent important treatment options for a number of conditions in future. AntagomiRs, for example, are able to suppress aberrantly expressed miRs; however, no therapies for rheumatic conditions have currently advanced from in vitro validation stages. While this line of investigation does not necessarily relate specifically to methylation profiling to predict treatment sensitivity, it demonstrates that other epigenetic mechanisms can be manipulated by treatment in order to manage a variety of diseases.

\section{Future perspective}

Studies which highlight epigenetic modifications associated with response to treatment not only possess enormous clinical utility in terms of optimizing patient care, but may also aid in reducing the economic burden associated with unnecessary drug prescriptions. As suggested, future research should strive to expand upon studies such as those conducted by Glossop et al.; collaboration between institutions could significantly assist in expanding study numbers, thereby improving statistical robustness of data. Also, similar studies which potentially highlight the influence of DNA methylation on treatment response in other conditions could have a positive impact on a much larger number of patients. Finally, once studies such as this are vali- 
dated in larger cohorts, it is essential that standardized procedures for measuring markers across different genomic sites are implemented.

\section{Financial \& competing interests disclosure}

The authors have no relevant affiliations or financial involvement with any organization or entity with a financial

\section{References}

1 Neidhart M, Rethage J, Kuchen S et al. Retrotransposable L1 elements expressed in rheumatoid arthritis synovial tissue: association with genomic DNA hypomethylation and influence on gene expression. Arthritis Rheum. 43(12), 2634-2647 (2000).

2 Karouzakis E, Rengel Y, Jungel A et al. DNA methylation regulates the expression of CXCL12 in rheumatoid arthritis synovial fibroblasts. Genes Immun. 12(8), 643-652 (2011).

3 Nakano K, Whitaker JW, Boyle DL, Wang W, Firestein GS. DNA methylome signature in rheumatoid arthritis. Ann. Rheum. Dis. 72(1), 110-117 (2013).

4 Nile CJ, Read RC, Akil M, Duff GW, Wilson AG. Methylation status of a single CpG site in the IL6 promoter is related to IL6 messenger RNA levels and rheumatoid arthritis. Arthritis Rheum. 58(9), 2686-2693 (2008).

5 Bui C, Barter MJ, Scott JL et al. cAMP response elementbinding (CREB) recruitment following a specific $\mathrm{CpG}$ demethylation leads to the elevated expression of the matrix metalloproteinase 13 in human articular chondrocytes and osteoarthritis. FASEB J. 26(7), 3000-3011 (2012).

6 Heyn H, Esteller M. DNA methylation profiling in the clinic: applications and challenges. Nat. Rev. Genet. 13(10), 679-692 (2012).

7 Reynolds GP, Fachim HA. Does DNA methylation influence the effects of psychiatric drugs? Epigenomics 8(3), 309-312 (2016).

8 Glossop JR, Nixon NB, Emes RD et al. DNA methylation at diagnosis is associated with response to disease-modifying drugs in early rheumatoid arthritis. Epigenomics doi:10.2217/ epi-2016-0042 (2016) (Epub ahead of print).

9 Farragher TM, Lunt M, Fu B, Bunn D, Symmons DP. Early treatment with, and time receiving, first disease-modifying antirheumatic drug predicts long-term function in patients interest in or financial conflict with the subject matter or materials discussed in the manuscript. This includes employment, consultancies, honoraria, stock ownership or options, expert testimony, grants or patents received or pending, or royalties.

No writing assistance was utilized in the production of this manuscript.

with inflammatory polyarthritis. Ann. Rheum. Dis. 69(4), 689-695 (2010).

10 Barres R, Yan J, Egan B et al. Acute exercise remodels promoter methylation in human skeletal muscle. Cell Metab. 15(3), 405-411 (2012).

11 Lane SC, Camera DM, Lassiter DG et al. Effects of sleeping with reduced carbohydrate availability on acute training responses. J. Appl. Physiol. (1985) 119(6), 643-655 (2015).

12 O'Reilly S. MicroRNAs in fibrosis: opportunities and challenges. Arthritis Res. Ther. 18, 11 (2016).

13 Altorok N, Tsou PS, Coit P, Khanna D, Sawalha AH. Genome-wide DNA methylation analysis in dermal fibroblasts from patients with diffuse and limited systemic sclerosis reveals common and subset-specific DNA methylation aberrancies. Ann. Rheum. Dis. 74(8), 1612-1620 (2015).

14 Varga J, Pasche B. Transforming growth factor beta as a therapeutic target in systemic sclerosis. Nat. Rev. Rheumatol. 5(4), 200-206 (2009).

15 Gras C, Ratuszny D, Hadamitzky C, Zhang H, Blasczyk R, Figueiredo C. miR-145 contributes to hypertrophic scarring of the skin by inducing myofibroblast activity. Mol. Med. 21, 296-304 (2015).

16 Ciechomska M, O'Reilly S, Suwara M, Bogunia-Kubik K, van Laar JM. MiR-29a reduces TIMP-1 production by dermal fibroblasts via targeting TGF-beta activated kinase 1 binding protein 1 , implications for systemic sclerosis. PLoS ONE 9(12), e115596 (2014).

17 Maurer B, Stanczyk J, Jungel A et al. MicroRNA-29, a key regulator of collagen expression in systemic sclerosis. Arthritis Rheum. 62(6), 1733-1743 (2010).

18 Kurowska-Stolarska M, Alivernini S, Ballantine LE et al. MicroRNA-155 as a proinflammatory regulator in clinical and experimental arthritis. Proc. Natl Acad. Sci. USA 108(27), 11193-11198 (2011). 